\title{
Kinetic Inductance Detectors For Future Observations of the Cosmic Microwave Background
}

\author{
Ryan William LeFebre \\ Department of Physics, University of Wisconsin-Madison \\ Madison, Wisconsin, USA
}

\begin{abstract}
Kinetic inductance detectors (KIDs) have a lot of potential in the field of astronomical observations; they are easy to fabricate and are highly multiplexible. KIDs can be as simple as a single lithographic layer and 1000s of them can be read out on a single line. Future observations of the cosmic microwave background (CMB) will require huge pixel count detector arrays and KIDs are able to fill this role promisingly. This paper primarily discusses the design and simulation of KIDs in Sonnet to be used in a polarimeter chip intended for observation of the polarization anisotropy of the CMB. Both background information of the CMB and KID theory will be reviewed.
\end{abstract}

\section{Introduction}

1.1. Scientific Motivation The universe is filled with a cosmic microwave background. This background is electromagnetic radiation that peaks in the microwave region. It was first accidentally discovered by Arno Penzias and Robert Wilson at Bell Labs in 1964. Ever since then the CMB has been fundamental to observational cosmology. The CMB provides important information about the early universe and provides evidence for the Big Bang Theory. The microwave radiation that fills the universe today can be explained as a relic of the time when the universe was a hot and opaque plasma. When the universe became transparent its temperature was around 3000 Kelvin. The temperature of the CMB today is around 2.7 Kelvin (Ryden, 2003); this decrease in temperature is due to the expansion of the universe.

There are three underlying problems in the standard Hot Big Bang Theory; these are the flatness problem, the horizon problem, and the monopole problem. All three problems are solved with the idea of inflation. Inflation was first proposed by Alan Guth in 1981 and in a cosmological context, inflation can generally be described as the exponential expansion of space in the very early universe (Ryden, 2003). This exponential expansion is thought to have caused gravitational waves which are thought to have left a distinct polarization in the CMB. If these polarizations could be detected then definitive evidence of inflation in the early universe could resolve unsolved problems and constrain new physics but detection is not easy (Krauss, 2010). This polarization anisotropy offers a significant measurement challenge because it is expected to be at least three orders of magnitude smaller than the temperature anisotropy (Lowitz, 2017).

1.2. CMB Detectors Detecting the polarization anisotropy in the CMB requires very precise measurements. Since the sensitivity of modern CMB dedicated telescopes is determined by photon arrival statistics, the total sensitivity is of an instrument is determined by the total number of detectors it has. It is predicted that on the order of 500,000 detectors will be needed to meet the requirements of detected the polarization anisotropy in the CMB (Abitbol, 2017).

There are three kinds of detectors used in CMB experiments today. They are coherent detectors, thermal bolometric detectors, and direct detectors. The leading contender for detectors in CMB experiments for the past decade has been transition edge sensors (TESs). TESs are a type of cryogenic bolometric detector, they are well understood and almost background limited. While TESs have performed admirably in the past, they may be ill suited for future CMB observations. TESs have a complex multi-layer fabrication process and have many readout wires per detector. Their yield and scalability make them problematic in large detector arrays. Kinetic inductance detectors are a type of direct detector and are well suited for future CMB observations. Where TESs struggle, KIDs shine. KIDs are easy to fabricate and can be as simple are one lithographic layer. 1000s of KIDs can also be read out on a single line using frequency-domain multiplexing (Zmuidzinas, 2012). KIDs fit the bill in the need for huge detector arrays. 
1.3. Kinetic Inductance Detectors KIDs are superconducting microresonators that utilize changes in their surface impedance to detect photons. A circuit equivalence of a KID can be seen in Figure 1 (b). They are a promising detector technology that was invented at Caltech and JPL in the early 2000s. Their operation principle is based on the concept of kinetic inductance. Pairs of electrons, called Cooper pairs (CPs), are the primary charge carriers in superconducting metals. CPs are bound together by an electron-phonon interaction of energy $2 \Delta$. They have zero DC resistance but exhibit a non zero AC impedance due to their inertial mass. This impedance due to the CPs changing velocity acts like an inductance, hence the name kinetic inductance.

The surface impedance of a superconducting metal, $Z_{s}$, is a sum of two terms

$$
Z_{s}=R_{s}+j \omega L_{s}
$$

The $R_{s}$ term describes losses due to the small fraction of unpaired electrons call quasiparticles (QPs). The $L_{s}$ term is an inductive term and includes the kinetic inductance from CPs. An incident photon with energy $h f>2 \Delta$ can
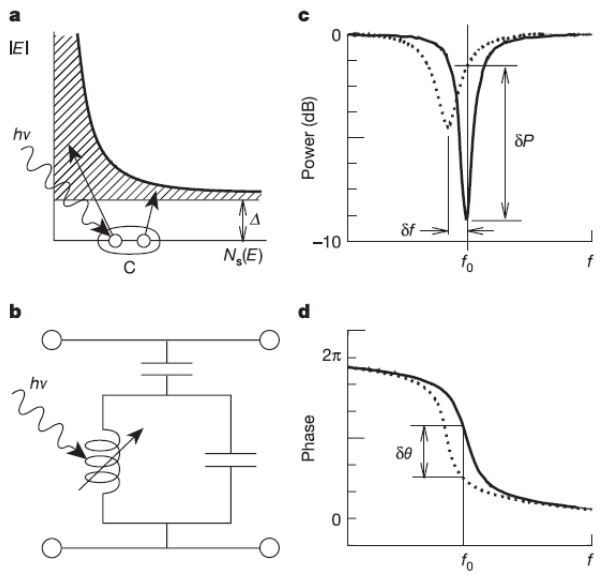

Figure 1: A depiction of KID detection principles (Mazin, 2004)

break CPs into QPs, Figure 1 (a). The change in number density of CPs and QPs results in a change of $L_{s}$ and $R_{s}$. Subsequently a change in the phase and resonant peak of the resonator as seen (c) and (d) of Figure 1 takes place. These changes can be read out by a probe line coupled to the KID.

1.4. Sonnet EM Analysis Software Sonnet is a high frequency electromagnetic software program that provides analysis and simulations of a variety of circuits and antennas. It is incredibly useful in designing and simulating kinetic inductance detectors. The project editor in Sonnet is a user friendly interface that allows you to create circuit geometries for subsequent analysis. Sonnets translators make it extremely versatile. Sonnet provides bidirectional translation of GDSII, DXF, and Gerber layout files to and from the project editor. Once a circuit is designed in or translated into the project editor it can be analyzed by Sonnets em electromagnetic analysis engine. Em performs a true three dimensional current analysis of predominantly planar structures by using a modified method of moments analysis based on Maxwells equations. The response viewer is Sonnets plotting tool. It allows you to plot your response data from em as a Cartesian graph or a Smith chart. A variety of data types can be plotted; they include the S, Y, and Z parameters, VSWR, Zin, Loss Factor, GMax, and Port Eeff. The current density viewer in Sonnet allows for qualitative views of the electromagnetic interactions occurring within the analyzed circuit. Radiation patterns of planar circuits and also be computed and displayed using Sonnets far field viewer. The far field viewer computes the far field radiation pattern of the analyzed circuit using the current density information from the em analysis. The far field Viewer can display plots in Cartesian, polar, or surface coordinates. Lastly Sonnet has a broadband Spice extraction module. This module generates equivalent lumped element circuits that can be used as an input in PSpice or Spectre (Sonnet, 2015). 


\section{Kinetic Inductance Detectors}

2.1. Advantages and Performance As already stated KIDs are simple to fabricate. Unlike TESs they do not require Josephson junctions or tuned bilayers. Fabricating large uniform arrays with KIDs is no problem. They are tremendously versatile in their design and implementation in microwave circuit layouts. High Q resonators also allow for passive frequency domain multiplexing of thousands of detectors through a single coaxial cable and single HEMT amplifier (Mazin, 2004).

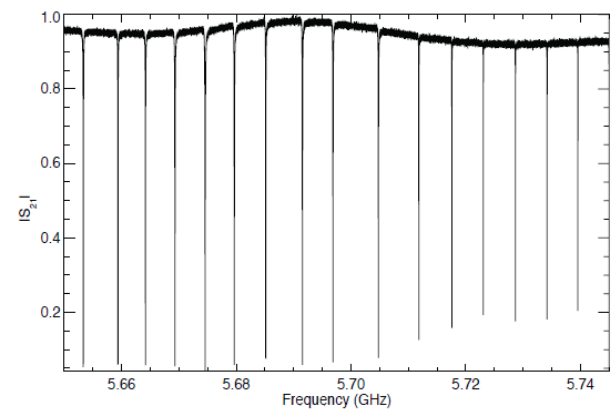

Figure 2: Frequency domain multiplexed KID resonators. Every peak is due to an individual KID (astrobites.org, 2011)

1000 pixel TES arrays have been built which approach background limited sensitivity for both ground and space based observations. KID arrays that have been built have not yet reached the same sensitivity levels but research in the area is ongoing and things look optimistic. A thorough examination of a comparison of noise properties in TESs and KIDs can be found in (Lowitz, 2017). There are two main fundamental noise sources in KIDs:

1. Photon Noise: Photon noise arises from astrophysical sources or backgrounds under observation.

2. Generation-Recombination Noise: This noise is caused by optical or thermal excitations that cause fluctuations in the number density of quasiparticles.

Presently, non-fundamental noise sources limit KIDs. These include two-level system noise in dielectric substrates but progress on tackling these problems is being made (Lowitz, 2017).

2.2. Superconductivity The operation of KIDs depends on superconductivity, a phenomenon first discovered in 1911 by Dutch physicist Heike Kamerlingh Onnes. Its hallmarks include zero DC resistance and expulsion of magnetic fields from the interior of the superconductor. These two properties take place at a temperature below the superconductor's transition temperature, $T_{c}$. In 1957 Bardeen, Cooper, and Schrieffer developed a microscopic theory of superconductivity (BCS theory). Once below the transition temperature electrons in the superconductor start to pair up into Cooper pairs. At temperatures well below $T_{c}$ near absolute zero, the binding energy of the CPs is

$$
\Delta(0) \approx 1.764 k_{B} T_{c}
$$

where $k_{B}$ is the Boltzmann constant. At temperatures just below the transition temperature the binding energy is (Zmuidzinas, 2012)

$$
\Delta(T) \approx 1.764 \Delta(0)\left(1-\frac{T}{T_{c}}\right)^{\frac{1}{2}}
$$

When analyzing the response of materials to alternating electric fields it is convenient to work with complex quantities and in this case the complex conductivity, $\sigma=\sigma_{1}+i \sigma_{2}$. In the limits of $k_{B} T \ll \Delta(0)$ and $\hbar \omega \ll \Delta(0)$ the BCS theory relates the real and imaginary parts of the complex conductivity to its value just above the transition temperature, $\sigma_{n}$, in the following ways:

$$
\frac{\sigma_{1}}{\sigma_{n}} \approx \frac{4 \Delta(T)}{\hbar \omega} \exp \left(-\frac{\Delta(0)}{k_{B} T}\right) K_{0}\left(\frac{\hbar \omega}{2 k_{B} T}\right) \sinh \left(\frac{\hbar \omega}{2 k_{B} T}\right)
$$


and

$$
\frac{\sigma_{2}}{\sigma_{n}} \approx \frac{\pi \Delta(T)}{\hbar \omega}\left[1-2 \exp \left(-\frac{\Delta(0)}{k_{B} T}\right) \exp \left(-\frac{\hbar \omega}{2 k_{B} T}\right) I_{0}\left(\frac{\hbar \omega}{2 k_{B} T}\right)\right]
$$

where $K_{0}$ and $I_{0}$ are modified Bessel functions (Giordano, 2009). For temperatures well below $T_{c}$, the BCS theory also gives an approximation for the density of quasiparticles, $n_{q p}$

$$
n_{p q} \approx 2 N(0) \sqrt{2 \pi_{B} T \Delta(0)} \exp \left(-\frac{\Delta(0)}{k_{B} T}\right)
$$

where $N(0)$ is the density of states at the Fermi surface (Giordano, 2009).

In these limits the surface impedance, $Z_{s}$ of a superconductor can be related to $\sigma$. For a very thin film,

$$
Z_{s} \approx \frac{1}{\left(\sigma_{1}+i \sigma_{2}\right) d}
$$

or

$$
R_{s} \approx \frac{\sigma_{1}}{\left(\sigma_{1}^{2}+\sigma_{2}^{2}\right) d}
$$

and

$$
\omega L_{s} \approx \frac{\sigma_{2}}{\left(\sigma_{1}^{2}+\sigma_{2}^{2}\right) d}
$$

Here, $d$ is the thickness of the thin film (Gao, 2008). The above equations show that when a photon breaks a Cooper pair and changes the quasiparticles density, the complex conductivity changes and subsequently the surface impedance is changed.

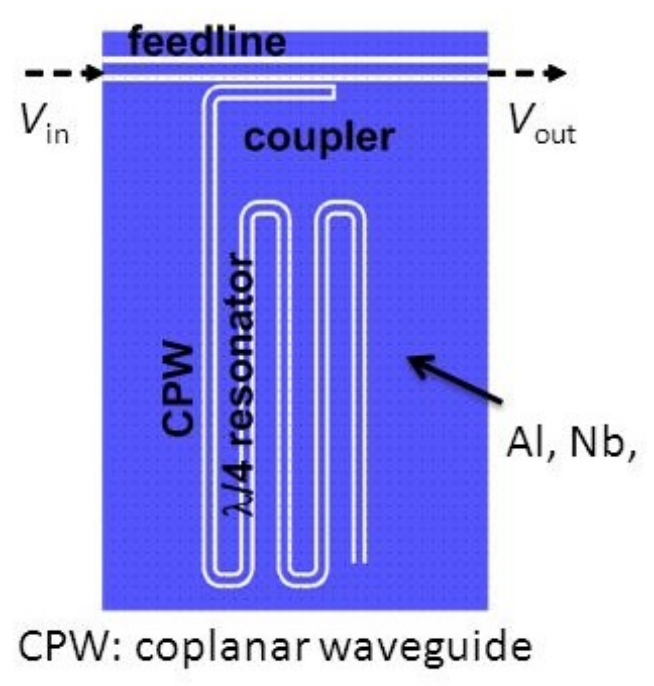

(a) Coupled Quarter Wave KID

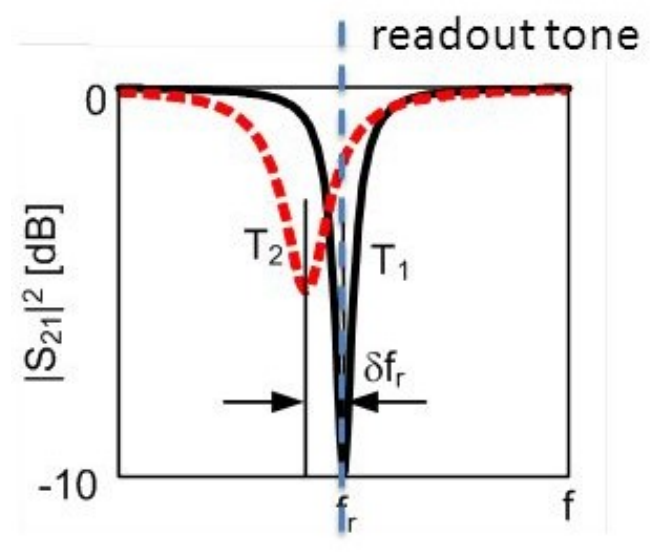

(b) Resonance Dip

Figure 3: Quarter wave KID coupled to a feedline with corresponding resonance dip (NIST)

2.3. KID Theory and the Half Wave Resonator KIDs are planar microwave resonators, often microstrip or coplanar waveguide (CPW), coupled to a feedline. As microwave resonators, they can easy be described by transmission line analysis. Scattering parameters are used to read the response of a KID. A signal is sent down the feedline and if it is near the resonant frequency of the resonator, some of it will be drawn into the resonator. This will create a distinct dip in the $S 21$ parameter, figure 3 (b). When the surface impedance of the KID resonator changes, a change in its scattering parameter will be seen. In a two port system, $S 21$ is equivalent to the transmission coefficient (Pozar, 2012)

$$
S 21=T=1+\Gamma=1+\frac{Z_{L}-Z_{0}}{Z_{L}+Z_{0}}=\frac{2 Z_{L}}{Z_{L}+Z_{0}} .
$$


Here $Z_{0}$ is the characteristic impedance of the feedline and $Z_{L}$ is the load attached to it. For the situation with a resonator capacitively coupled to a feedline,

$$
\frac{1}{Z_{L}}=\frac{1}{Z_{\text {in }}^{\text {coupledres }}}+\frac{1}{Z_{0}^{\text {feedline }}}
$$

and now

$$
S 21=\frac{2 Z_{\text {in }}^{\text {coupledres }}}{2 Z_{\text {in }}^{\text {coupledres }}+Z_{0}^{\text {feedline }}} .
$$

Equation 12 shows how the response of a resonator changes with a change in $Z_{\text {in }}^{\text {coupledres }}$.

It is often helpful when dealing with transmission line resonators to equate them to equivalent discrete element circuits.

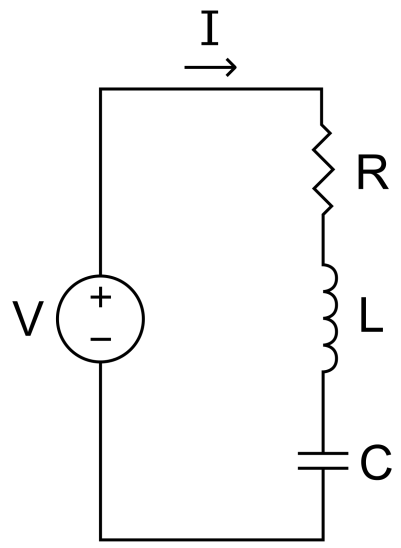

(a) Series RLC Circuit

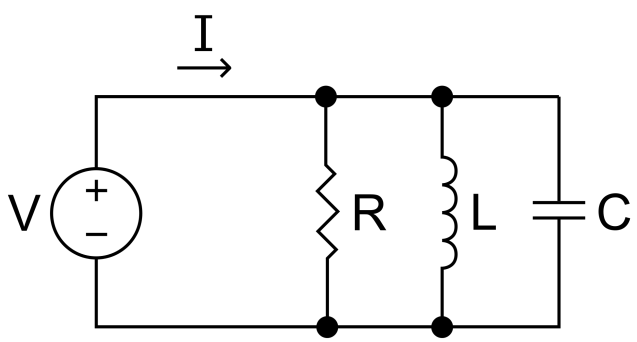

(b) Parallel RLC Circuit

Figure 4: Discrete element RLC circuits

The impedance and quality factors of a series and parallel RLC circuit near its resonant angular frequency, $\omega_{0}$, are

$$
\begin{gathered}
Z_{\text {in }}^{\text {series }}=\frac{\omega_{0} L}{Q_{i}^{\text {series }}}+j 2 L\left(\omega-\omega_{0}\right)=R_{i}+j 2 L \Delta \omega \\
Q_{i}^{\text {series }}=\frac{\omega_{0} L}{R_{i}} \\
Q_{L}^{\text {series }}=\frac{\omega_{0} L}{R_{L}}
\end{gathered}
$$

and

$$
\begin{gathered}
Z_{\text {in }}^{\text {parallel }}=\frac{R}{1+2 j \Delta \omega R C}=\frac{R}{1+2 j Q_{0} \Delta \omega / \omega_{0}} \\
Q_{i}^{\text {parallel }}=\frac{R_{i}}{\omega_{0} L} \\
Q_{L}^{\text {parallel }}=\frac{R_{L}}{\omega_{0} L}
\end{gathered}
$$


here $j$ is the imaginary number, $R_{i}$ is the internal resistance, $R_{L}$ is the load resistance, $Q_{i}$ is the internal quality factor, and $Q_{L}$ is the loaded quality factor (Pozar, 2012).

The half wave resonator is an open circuited transmission line whose length is one half the wave length of its corresponding resonant frequency

$$
l=\frac{\lambda}{2}=\frac{v_{p}}{2 f}=\frac{1}{2 f \sqrt{L_{l} C_{l}}} .
$$

In this equation $L_{l}$ and $C_{l}$ are the resonator's inductance per length and capacitance per length respectively (Pozar, 2012). For a superconducting thin film, $L_{l}$ is the surface inductance per length, which includes a kinetic term. This equation shows that when Cooper pairs are broken, the resonant frequency of a resonator is lowered.

The impedance of a capacitively coupled open ended half wave resonator is derived in (Pozar, 2012):

$$
Z_{\text {in }}^{\text {coupledres }}=\frac{\pi}{2 Q_{i}^{r e s} Z_{0}^{r e s} \omega^{2} C_{c}^{2}}+\frac{j \pi \Delta \omega}{\omega_{0} Z_{0}^{r e s} \omega^{2} C_{c}^{2}}
$$

In this equation $C_{c}$ is the coupling capacitance between the resonator and feedline. Equation 20 looks just like a series discrete element resonator and if we match the terms we can find its quality factors

$$
\begin{gathered}
R_{i}^{\text {coupledres }}=\frac{\pi}{2 Q_{i}^{\text {res }} Z_{0}^{\text {res } \omega^{2} C_{c}^{2}}} \\
L^{\text {coupledres }}=\frac{\pi}{2 \omega_{0} Z_{0}^{\text {res } \omega^{2} C_{c}^{2}}} \\
Q_{i}^{\text {coupledres }}=\frac{\omega_{0} L^{\text {coupledres }}}{R_{i}^{\text {coupledres }}}=Q_{i}^{\text {res }} \\
Q_{c}^{\text {coupledres }}=\frac{\omega_{0} L^{\text {coupledres }}}{R_{\text {Load }}}=\frac{\pi}{2 Z_{0}^{\text {res } \omega^{2} C_{c}^{2} R_{L o a d}}=\frac{\pi}{Z_{0}^{r e s} \omega^{2} C_{c}^{2} Z_{0}^{\text {feedline }}}} \\
Q_{\text {total }}^{\text {coupledres }}=\left(\frac{1}{Q_{i}^{\text {coupledres }}}+\frac{1}{Q_{c}^{\text {coupledres }}}\right)^{-1}=\frac{\pi Q_{i}^{\text {res }}}{\pi+Z_{0}^{\text {res }} Z_{0}^{\text {feedline }} \omega^{2} C_{c}^{2}}
\end{gathered}
$$

In equation 24 , the resonator see the feedline as a load of two impedances in parallel so $R_{\text {Load }}=\frac{1}{2} Z_{0}^{\text {feedline }}$. Knowing the quality factors, it is possible to rewrite the impedance of the coupled resonator and in turn the scattering parameter S21.

$$
\begin{gathered}
Z_{\text {in }}^{\text {coupledres }}=\frac{Q_{c}^{\text {coupledres }} Z_{0}^{\text {feedline }}}{2 Q_{i}^{\text {coupledres }}}+j Q_{c}^{\text {coupledres }} Z_{0}^{\text {feedline }} \frac{\Delta \omega}{\omega} \\
S 21=\frac{\frac{Q_{c}^{\text {coupledres }}}{Q_{i}^{\text {coupledres }}}+j 2 Q_{c}^{\text {coupledres }} \frac{\Delta \omega}{\omega}}{\frac{Q_{c}^{\text {coupledres }}}{Q_{i}^{\text {coupledres }}}+1+j 2 Q_{c}^{\text {coupledres }} \frac{\Delta \omega}{\omega}}=1-\frac{\frac{Q_{\text {total }}^{\text {coudres }}}{Q_{c}^{\text {coupledres }}}}{1+j 2 Q_{\text {toutal }}^{\text {coupledres }} \frac{\Delta \omega}{\omega}}
\end{gathered}
$$

It has been shown $(\mathrm{Gao}, 2008)$ that the response of a resonator is maximized when $Q_{c}=Q_{i}$. This is an important design consideration to keep in mind. This fact and equation 27 tell us that on resonance

$$
S 21=1-\frac{Q_{\text {total }}^{\text {coupledres }}}{2 Q_{\text {total }}^{\text {coupleses }}}=\frac{1}{2} .
$$


2.4. Half Wave Resonator with Input into Virtual Ground When designing kinetic inductance detectors it is advantageous to have a high total quality factor, to maximize responsivity, and to minimize noise. Figure 5 below shows a microstrip half wave KID designed and simulated in Sonnet. The KID is comprised of two metals. Niobium is represented as green and the resonator's quasiparticle trap is shown in red. The trap is made out of manganese doped aluminum. The resonator is approximately $11.5 \mathrm{~mm}$ long, its trap totals $3 \mathrm{~mm}$ in length. The resonator is a uniform $6 \mu \mathrm{m}$ wide and the total area covered by it is $540 \mu \mathrm{m} \times 690 \mu \mathrm{m}$. The advantage of this sort of design is that radiation is coupled into the resonator (coming from port 3 in Figure 5) on the same plane that the resonator exists on. This works because a half wave resonator has a virtual ground at its center and a microstrip line tapping into it does not affect the integrity of its resonance.

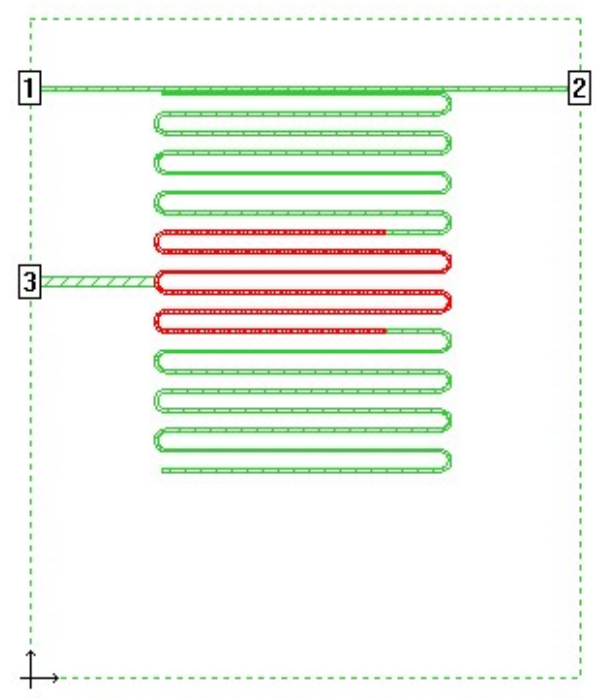

Figure 5: Hybrid $\mathrm{Nb}$ and AlMn Half Wave Microstrip Resonator

Figure 6 (a) shows that the hybrid Nb/AlMn half wave resonator has a resonant frequency around $4 \mathrm{GHz}$. The resonant peak is very deep and measuring the bandwidth at half its maximum allows for its total quality factor to be measured

$$
Q_{\text {total }}^{\text {coupledres }}=\frac{f_{0}}{\Delta f}
$$

Here $f_{0}$ is the resonant frequency. Using Figure 6 (b) it can be seen that the total quality factor of the resonator in figure 5 is about 5000 .

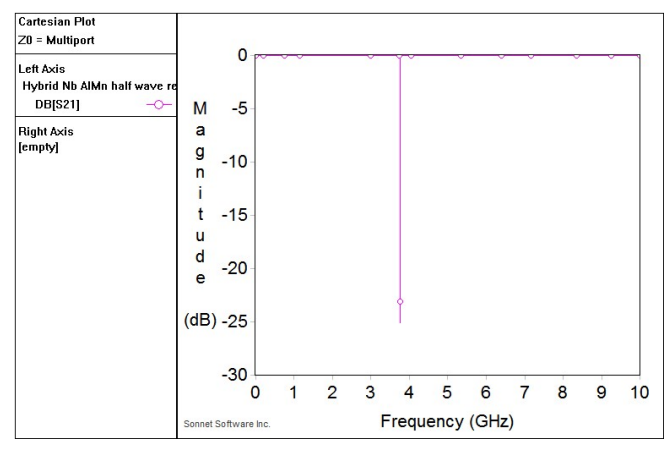

(a) Resonance Peak

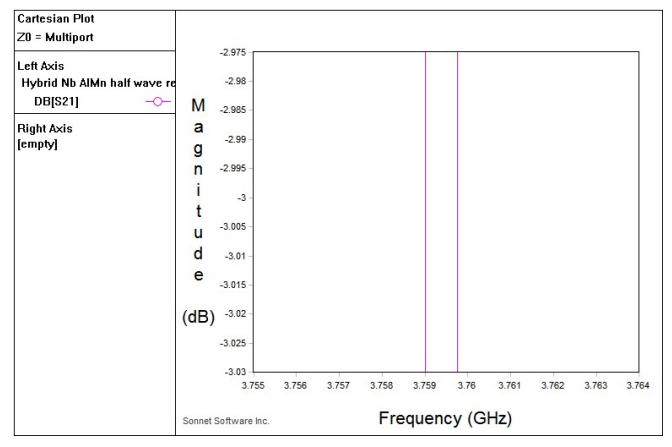

(b) Zoomed In

Figure 6: The Resonance Peak of the Microstrip Half Wave Resonator

The role of the quasiparticle trap is to absorb the incoming radiation and keep the generated quasiparticles within it. 
Manganese doped aluminum is fairly lossy and using $0.5 \Omega / s q$ in Sonnet gave decent results. Figure 7 (b) shows that around 90 percent of the power entering through port 1 is absorbed by the trap at frequencies higher than $40 \mathrm{GHz}$.

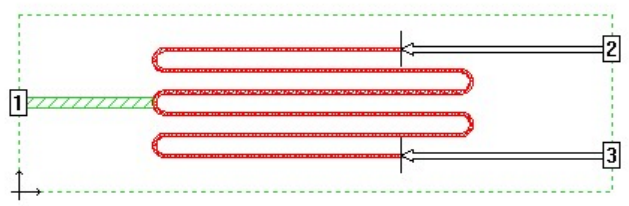

(a) Quasiparticle Trap

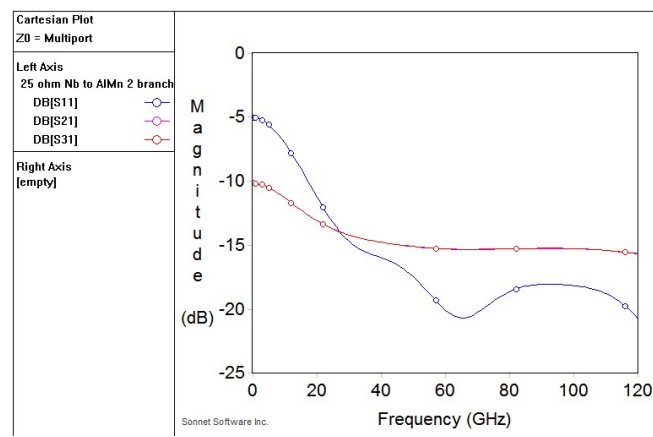

(b) S Parameters

Figure 7: Quasiparticle Trap and Corresponding S Parameters

\section{Conclusion and Future Work}

There is a lot of work to be done in the near future. The half wave resonator's $S 21$ parameters are not optimized. More simulations need to be run to get $Q_{i}=Q_{c}$ or correspondingly $S 21=\frac{1}{2}$. Optimizing $S 21$ should also help raise its total quality factor, which is not that high right now. It would also be ideal to have the trap on the resonator absorb 100 percent of the power going into it.

In the future, the eventual goal is to construct a polarimeter chip utilizing KIDs. Figure 11 shows a depiction of a polarimeter chip used in CLASS (Chuss, 2015). There are many planar microwave components involved in a polarimeter chip besides the detectors. They include an orthomode transducer (OMT), cross-overs, magic tees or hybrid couplers, and broadband filters. With the exception of the OMT all of these components can be designed and simulated in Sonnet. It is also worth looking into alternative components to be use in a polarimeter chip. For instance, if a dual-polarized antenna such as the one in (Yahya, 2011) could replace the OMT then there would be no need for a cross-over or hybrid couplers. This would simplify the polarimeter chip.

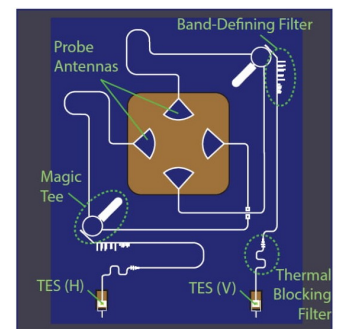

Figure 8: Depiction of a Polarimeter Chip (Chuss, 2015)

\section{Acknowledgments}

I would like to thank Peter Timbie and the rest of the Observational Cosmology Group as well as the National Space Grant College and Fellowship Program and the Wisconsin Space Grant Consortium for making this opportunity possible. 


\section{References}

Abitbol, Maximilian H. CMB-S4 Technology Book, First Edition. 2017, doi:10.2172/1414402.

Chuss, David T., et al. Cosmology Large Angular Scale Surveyor (CLASS) Focal Plane Development. Journal of Low Temperature Physics, Nov. 2015.

Gao, Jiansong. The Physics of Superconducting Microwave Resonators. California Institute of Technology, 2008.

Giordano, Claudia. MKID Arrays: Panoramic Detectors for CMB Experiments. Sapienza University of Rome, 2009.

https://astrobites.org/2011/12/06/microwave-kinetic-inductance-detectors/

Krauss, Lawrence. Primordial Gravitational Waves and Cosmology. Apr. 2010.

Lowitz, Amy E. Kinetic Inductance Detectors for CMB Polarimetry at $100 \mathrm{GHz}$. University of Wisconsin-Madison, 2017.

Mazin, Benjamin. ’Microwave Kinetic Inductance Detectors.” California Institute of Technology, 2004

NIST Quantum Devices Group, Boulder, CO

Pozar, David M. Microwave Engineering. 4th ed., Wiley, 2012.

Ryden, Barbara. Introduction to Cosmology. 1st ed., Addison Wesley, 2003.

Sonnet Software, Inc. ”Sonnet User's Guide.” North Syracuse, NY, October 2015

Yahya, R., and T. A. Denidni. Design of a New Dual-Polarized Ultra-Wideband Planar CPW Fed Antenna. 2011 IEEE International Symposium on Antennas and Propagation (APSURSI), 2011, doi:10.1109/aps.2011.5996837.

Zmuidzinas, Jonas. Superconducting Microresonators: Physics and Applications. Annual Review of Condensed Matter Physics, 2012 